\title{
Perspectives on the Role of Photodynamic Therapy in the Treatment of Pancreatic Cancer
}

\author{
Wei Li, ${ }^{1}$ Qingyong Ma, ${ }^{1}$ and Erxi $\mathrm{Wu}^{2}$ \\ ${ }^{1}$ Department of Hepatobiliary Surgery, First Affiliated Hospital of Medical College, Xi'an Jiaotong University, 277 West Yanta Road, \\ Xi'an 710061, Shaanxi Province, China \\ ${ }^{2}$ Department of Pharmaceutical Sciences, North Dakota State University, Sudro Hall 203, Fargo, ND 58105, USA
}

Correspondence should be addressed to Qingyong Ma, qyma56@mail.xjtu.edu.cn

Received 15 May 2011; Revised 25 July 2011; Accepted 1 August 2011

Academic Editor: Peter Robertson

Copyright () 2012 Wei Li et al. This is an open access article distributed under the Creative Commons Attribution License, which permits unrestricted use, distribution, and reproduction in any medium, provided the original work is properly cited.

Photodynamic therapy (PDT) is a noninvasive procedure involving a photosensitizing agent that is activated by light to produce reactive oxygen species (ROS) that selectively destroy tumor cells. In recent years, PDT has been used in the treatment of pancreatic cancer (PC). The antitumor effects of PDT include three main mechanisms: direct tumor cell death (necrosis, apoptosis, and autophagy), vascular destruction, and immune system activation. The present paper systematically summarizes the effects of PDT in the treatment of PC from the experimental studies to the clinical studies and discusses the mechanisms of PDT-induced PC destruction.

\section{Introduction}

Pancreatic cancer (PC) is one of the most lethal malignant diseases and has a dismal prognosis. It is estimated that over 37,000 patients were newly diagnosed with PC, and 34,000 patients died of this disease in the USA in 2010 [1]. PC has the lowest 5-year survival rate of any gastrointestinal tumor, and the median survival rate is no more than 6 months $[1,2]$. Surgery remains the only way to cure this disease, but less than $20 \%$ of patients are considered for surgical resection at the time of initial diagnosis [3]. Moreover, even seemingly resectable PC often fails to cure the disease due to the microscopic systemic spread of the cancer that occurred before the operation [4]. Current treatments for inoperable patients are still limited to chemotherapy, radiation, or both (chemoradiotherapy) [5]. A new comprehensive and constructive therapy is urgently needed.

Photodynamic therapy (PDT) is a treatment that uses nontoxic drugs or dyes (photosensitizers) that are pharmacologically active only after exposure to light in the presence of oxygen $[6,7]$. Due to its fundamental selectivity and specificity [8], PDT has been considered to be a possible treatment for neoplasms, including cancers of the skin [9], head and neck [10], nasopharynx [11], esophagus, lung [12], pancreas, biliary duct [13], and bladder [14]. The four main kinds of photosensitizers are porphyrin derivatives, chlorines, phthalocyanines, and porphycenes [7, 15] (Figure 1). The photosensitizer excited triplet state undergoes two broad kinds of reactions (Type I and Type II). In a Type I reaction, the triplet photosensitizer can transfer an electron to a neighboring substrate to form free radicals and radical ions, which further interact with molecular oxygen and produce reactive oxygen species (ROS). In a Type II reaction, the triplet photosensitizer can transfer its energy directly to molecular oxygen and form excited-state singlet oxygen [16, 17] (Figure 2). These two reactions constitute the core mechanism of PDTmediated destruction of tumor tissue. In recent years, more and more research has focused on the development of PDT for the treatment of PC [18].

\section{Experimental Studies}

2.1. Mechanisms of Pancreatic Cancer Cell Death in PDT. Cells can undergo three distinct kinds of cell death induced by PDT: necrosis, apoptosis, and autophagy [7, 19]. Necrosis is morphologically characterized by increased cellular volume, swelling of organelles, plasma membrane rupture and the subsequent loss of intracellular contents [20]. It is 


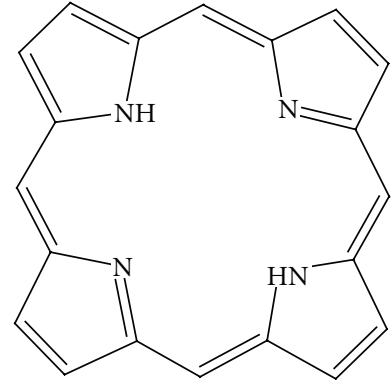

Porphyrin

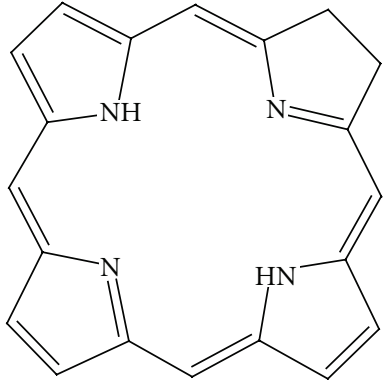

Chlorin

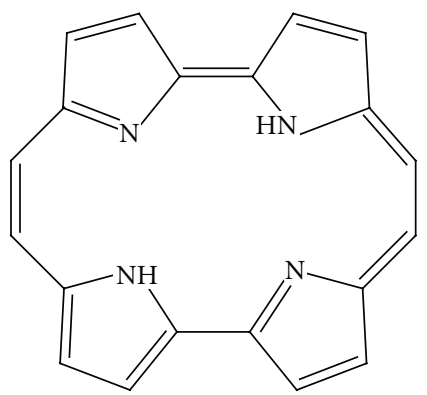

Porphycene

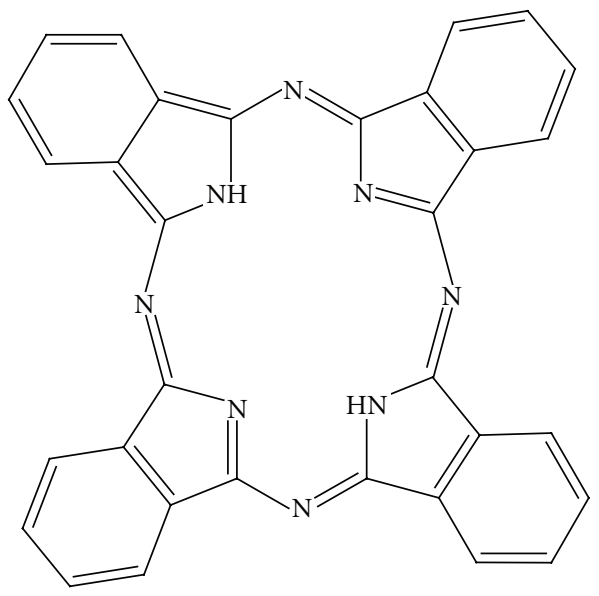

Phthalocyanine

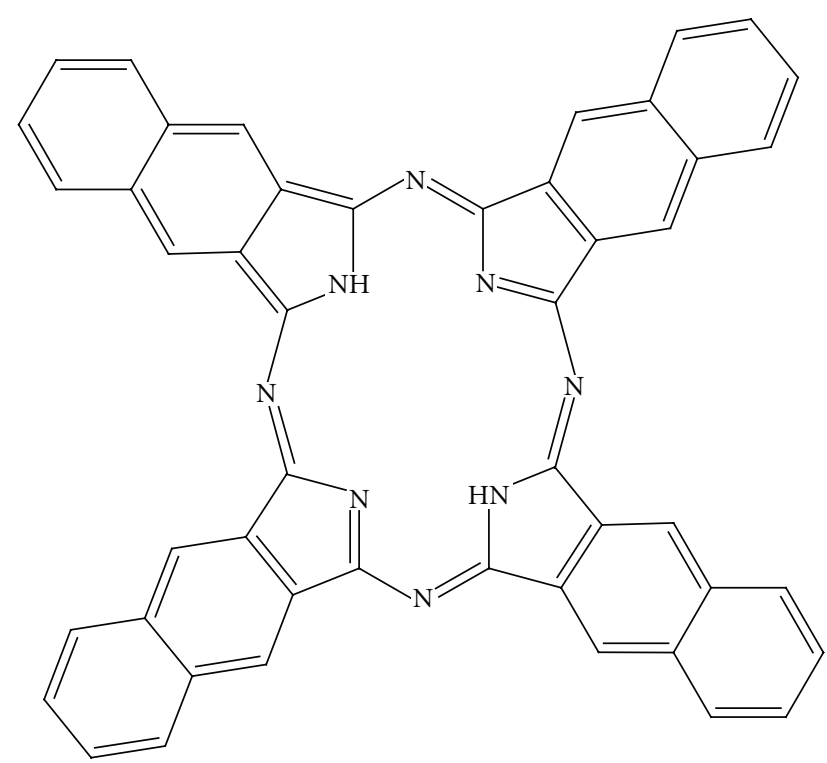

Naphthalocyanine

FIgURE 1: Chemical structures of major PDT photosensitizers.

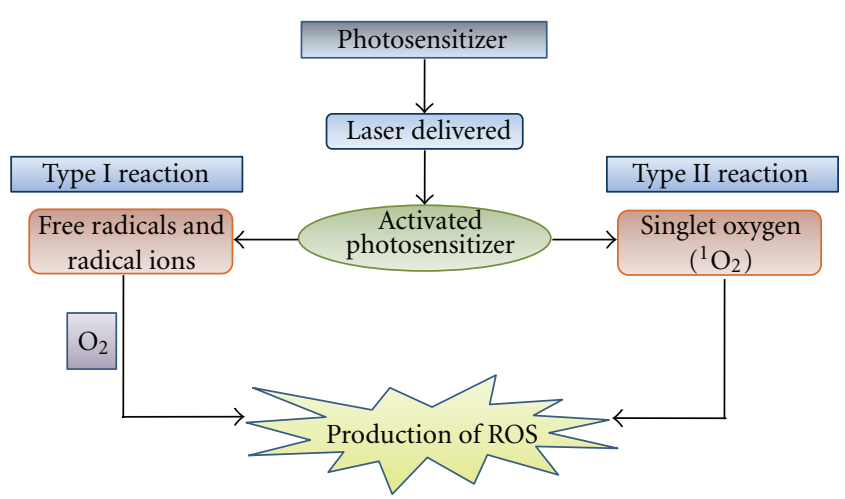

FIgure 2: Mechanisms leading to the formation of ROS. There are two types of reactions during PDT. In Type I reaction, the triplet photosensitizer reacts with the neighboring substrate and forms free radicals as well as radical ions, which further interact with molecular oxygen and produce ROS. In Type II reaction, the triplet photosensitizer transfers its energy directly to molecular oxygen and form excited-state singlet oxygen. generally believed that higher light dosage is always accompanied by cellular necrosis [21]. Using different orthotopic pancreas cancer xenograft models (AsPC-1 and Panc-1), Samkoe et al. [21] demonstrated that both Panc-1 tumors and AsPC-1 tumors became necrotic following treatment with verteporfin PDT and hematoxylin/eosin-stained tumor slices displayed increasing necrotic/edemic core with the increase of light dose. Besides, faster growing tumors (AsPC1 cell line) were relatively easier to treat.

Another in vivo experiment reported by Xie et al. [22] showed that PDT led to necrosis in cancer lesions and significantly reduced tumor volume. They observed that partial tumor necrotic tissue was exfoliated and a necrotic edge of volcano-like uplift was formed 1 week after PDT treatment. In a randomized, controlled study of implanted pancreatic cancers in Syrian golden hamsters treated with 5aminolaevulinic acid, PDT-induced tumor necrosis of up to $8 \mathrm{~mm}$ in depth was achieved, and the survival time of 
the treated animals was significantly longer than in the untreated control group [23].

Mlkvy et al. [24] conducted experiments to assess the effects of meta-tetrahydroxyphenylchlorin (mTHPC) in a hamster pancreatic cancer model. In their experiment, 0.1 or $0.3 \mathrm{mg} / \mathrm{kg}$ mTHPC was given to the animals, and the tumor was treated 2 or 4 days later via laparotomy with red light (50 J at $650 \mathrm{~nm}$, continuous or fractionated) delivered through a single fiber touching the tumor surface. The results showed that the zones of tumor necrosis, often haemorrhagic in the center, sharply demarcated from adjacent viable tumor with an inflammatory infiltrate in the surrounding area. This may be attributed to the vascular supply pattern of the tumor or the protective role of surrounding connective tissue strips dividing tumor into lobules $[23,24]$. The results also elucidated that the maximum zone of tumor necrosis was $8.7 \mathrm{~mm}$ in diameter with continuous irradiation, which increased to $12.4 \mathrm{~mm}$ with fractionated treatment.

In addition, PDT has also been proven to damage DNA. Hajri et al. [25] demonstrated that PDT inhibited tumor cell growth in cell culture by affecting DNA integrity. The DNAdamaging effects of PDT are related not only to variables in PDT but also to cellular repair and survival mechanisms [26]. Ferreira et al. [27] designed synthetic oligonucleotides (aptamers) that were selected to bind to unique short $\mathrm{O}$-glycan-peptide signatures on the surface of pancreatic cancer cells and observed a remarkable enhancement $(>500$ fold increase) in toxicity of PDT in the presence of these phototoxic aptamers.

Apoptosis, another type of cell death, requires ATP and is characterized by cytoplasmic shrinkage, reduction of cellular volume, condensation of the chromatin, and fragmentation of the nucleus [28]. Several pathways have been proven to play a role in cellular death. For example, the classic antiapoptotic proteins in the Bcl-2 family can be downregulated after PDT. It is known that $\mathrm{Bcl}-2$ is a molecular target of PDT using mitochondrion-targeting photosensitizers and can determine the sensitivity of cancer cells to apoptosis and the overall cancer response to PDT [29]. Lutetium-texaphyrinmediated PDT can not only downregulate the expression of $\mathrm{Bcl}-2$ and upregulate the expression of Bax in bovine retinal capillary endothelial cells, but also influence $\mathrm{Bcl}-\mathrm{xL}$ and Bak proteins in human retinal pigment epithelial cells [30]. Using phthalocyanine photosensitizer Pc4-mediated PDT, He et al. [31] found that parental cells displayed a high incidence of apoptosis after PDT, whereas Bcl-2-transfected cells exhibited a much lower incidence of apoptosis as assessed by DNA fragmentation. Another apoptosis-related protein, cytochrome c, was released from mitochondria upon treatment with PDT $[32,33]$. It is reported that the release of cytochrome $\mathrm{c}$ from mitochondria is controlled by proteins of the Bcl-2 family. Liu et al. [34] observed that cytochrome c was released from the mitochondria into the cytoplasm during PDT, and the mitochondria membrane potential $(\Delta \Psi \mathrm{m})$ showed a loss of nearly $30 \%$ in human pancreatic cancer cells. After releasing into the cytosol, cytochrome c is able to initiate apoptotic signal events, activating caspase- 9 and then caspase- 6 and caspase-7, respectively [35]. A second pathway involved in cell death stems from PDT-activated caspases and the subsequent cleavage of the DNA repair protein poly (ADP-ribose) polymerase [36]. Yet another pathway involves the Fas ligand (FasL), which belongs to the tumor necrosis factor (TNF) family. When FasL binds to its receptor, apoptosis is induced. Fas/FasL system could either signal the apoptosis directly through the activation of the caspase system or through mitochondria [37]. PDT has been proven to enhance FasL expression, leading to an increase in FasL signaling-dependent cell death in cancer cells. A recent study has shown that PDT induced apoptosis of nasopharyngeal, colon, and bladder cells is mediated not only by activation of Fas with the involvement of the FasL system, but also by the activation of a distinct caspase cascade [38]. The activation of the caspase cascade, caspase- 8 and caspase-3, follows direct activation of Fas/FasL in PDTinduced apoptosis [38]. Calcium plays an important role in photodynamic drug action. PDT-induced increases in the levels of intracellular calcium may be associated with cell apoptosis [7]. Calcium chelators were shown to inhibit the PDT-induced release of cytochrome $c$, caspase-3 activation and apoptosis in Chinese hamster V79 cells, indicating that calcium indeed plays a role in PDT-induced apoptosis [39]. In a recent study, Chiou et al. [40] showed that verteporfin PDT could rapidly provoke hyperoxidative stress and caspase activity in HepG2 cells. In addition, the membrane integrity was decreased and permeability increased, resulting in a sudden influx of cytosolic calcium into the mitochondria. All these factors were treated as the arbitrator to initiate the lethal apoptotic process after verteporfin PDT. The increase in the intracellular calcium concentration upon photosensitization may occur via the influx of calcium through ion channels, the release of calcium sequestered in internal stores and/or the activation of ion exchange mechanisms [41].

Cellular adhesion is also associated with cellular apoptosis. Galaz et al. [42] demonstrated that the loss of E-cadherinmediated cell adhesion after early photodamage triggers an apoptotic response. They also observed that the alteration in E-cadherin preceded the release of cytochrome $\mathrm{c}$ from the mitochondria to the cytosol as well as the activation of caspase 3. Blocking E-cadherin function with a specific antibody induced apoptosis. PDT can also downregulate the expression of vascular cell adhesion molecule- 1 and intracellular cell adhesion molecule-1 [43]. A notable feature of PDT is to change the attachment between cancer cells and stroma or cancer cells themselves, which can be attributed to the damage of adhesion molecules [44].

In recent years, more and more researchers have expressed concern about the p53-mediated cytotoxicity of the PDT of cancer [45]. A direct evidence supporting the idea that p53 is involved in PDT response came from the work by Mitsunaga et al. [46]. In their research, they showed that the activition of caspase- 3 and caspase- 9 increased in wild-type human colon cancer cells. In contrast, it was significantly inhibited in Bax-null or p53-null cells which indicated that the caspase-dependent apoptosis induced by PDT was Baxand p53-dependent. Lim et al. [47] evaluated the ability of PDT combined with a tumor suppressor factor, recombinant adenovirus p53 (AdCMVp53), to induce apoptosis as well as cell growth inhibition. They noticed that cotreatment with 


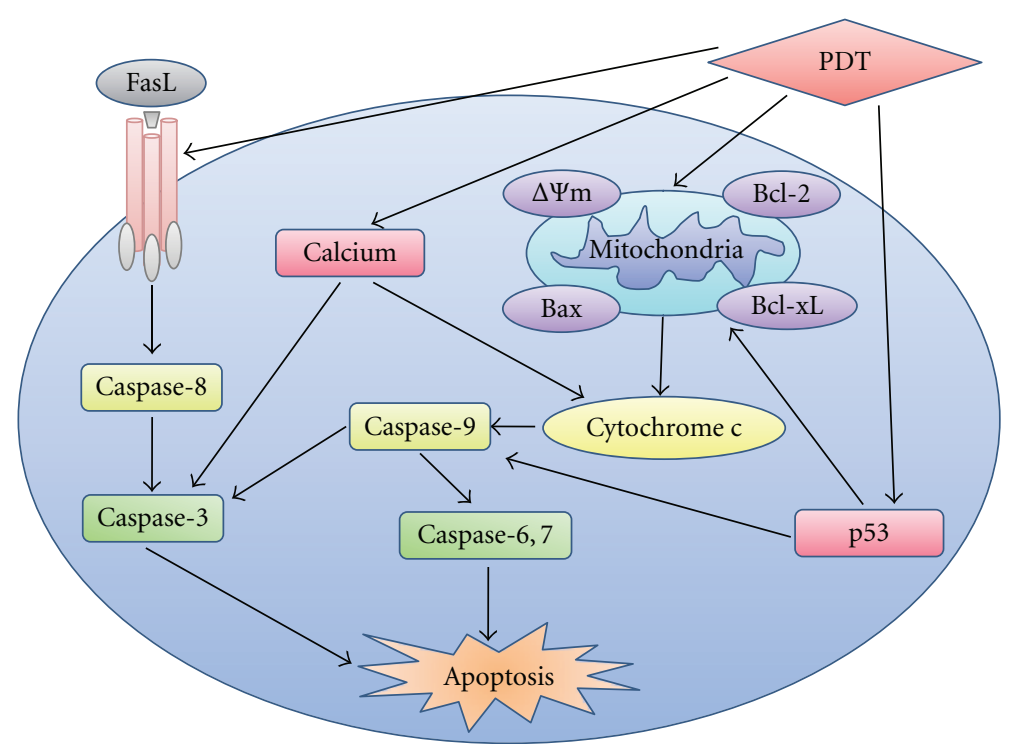

FIGURE 3: Schematic illustration represents the possible effect of PDT on the apoptosis pathways. PDT-associated apoptotic progress is a complicated occurrence which activates the mitochondrial pathway, promotes cytochrome $c$ releases and caspase-3, $-6,-7$ activation. Moreover, PDT also influences cancer cellular apoptosis via the elevation levels of p53 and intracellular calcium.

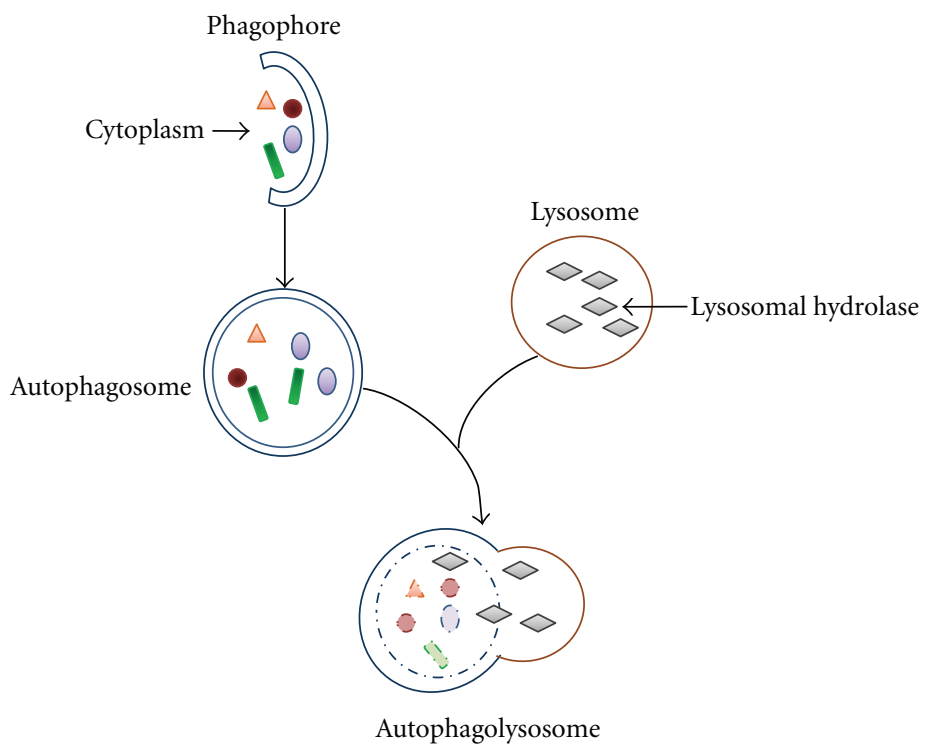

FIGURE 4: Schematic model of autophagy. First of all, a double membrane structure named autophagosome surrounds the target region and creates a vesicle which separates its contents from the rest of the cytoplasm. Secondly, the vesicle is transported and fused to the lysosome, forming autophagolysosome. Lastly, the contents are degraded by lysosomal hydrolases.

PDT and AdCMVp53 resulted in a more potent antitumor effect. Cotreatment led to elevated levels of p53, possibly causing the induction of p53-dependent apoptosis. It is generally believed that the key factors that determine the type of PDT-induced cell death are cell genotype, light dose, and the subcellular localization of the photosensitizers $[7,48]$ (Figure 3).

Autophagy is a process in which the abnormal cytoplasm is sequestered into double-membrane vesicles and fused by lysosomes, with the contents of the autophagosomes being digested and recycled $[49,50]$ (Figure 4). Due to the morphological and biochemical features of autophagic cell death, it is distinct from both apoptosis and necrosis [51]. Because autophagy develops in a sequential fashion, it is classified as a second type of programmed cell death. Both autophagy and apoptosis occur following PDT [19]. It is shown that autophagy is independent of photosensitizer target, because it is observed with photosensitizers localize in endoplasmic reticulum, mitochondria, lysosomes, and endosomes [52]. PDT can not only affect autophagy by damaging organelles 
(lysosomes and endosomes) but also influence proteins that are involved in this mechanism [53, 54]. Using shRNA technology, Kessel and Arroyo [55] created a Bax knockdown line. A marked decrease in apoptosis was observed after photodamage or pharmacologic inactivation of $\mathrm{Bcl}-2$ function in this cell line, but the PDT efficacy was not affected because the suppression of apoptosis leaded to enhanced autophagy (a highly vacuolated morphology). Autophagy appears to play a prosurvival role in apoptosis-competent cells and a prodeath role in apoptosis-incompetent cells [52].

\subsection{Mechanisms of Pancreatic Tumor Destruction}

2.2.1. Vascular Destruction. Pathological angiogenesis is a hallmark of tumor cells, and their viability depends on an adequate blood supply [56]. PDT-induced vascular damage is an important mechanism of tumor destruction. PDTmediated vascular effects range from transient vascular spasm, vascular stasis, and the formation of thrombus to permanent vessel occlusion [57]. Vascular destruction may contribute to a reduction in tissue oxygenation and further promote tumor destruction [58]. Li and Luo [59] showed that the antitumor effects of PDT were achieved mainly by the destruction of tumor blood vessels and the formation of thrombosis at short drug-light intervals; in contrast, the tumor cells were killed directly by PDT-mediated cytotoxicity at long drug-light intervals.

Although the oxygen-consuming reaction of PDT mediates the destruction of tumor vessels, the hypoxic condition within tumors can cause the release of angiogenic growth factors and cytokines that could possibly decrease the efficacy of PDT by promoting tumor regrowth [60]. Zhou et al. [61] demonstrated that the expression of hypoxia-inducible factor (HIF)-1alpha, vascular endothelial growth factor (VEGF), and cyclooxygenase 2 (COX-2) were increased in PDT-treated tumors, indicating that PDT-induced damage to tumor microvasculature and the resultant hypoxia upregulated the expression of certain proangiogenic factors. Combining antiangiogenesis inhibitors along with PDT led to greater efficacy in cancer treatment [62]. In addition, through a concept called "arterial flow focalization," which allows for controlled temporary vascular occlusion of the collateral arterial branches upstream of the tumor, it is possible to redirect blood flow through the principal artery of the downstream tumor, thereby increasing tumor arterial flow and hence oxygen supply, thus further greatly improving the efficacy of PDT [63].

2.2.2. Immune System Activation. PDT-induced necrosis of tumor cells with the subsequent induction of an inflammatory response leads to antitumor immune responses $[64,65]$. It has been reported that PDT alters the tumor microenvironment by stimulating the release or expression of various proinflammatory and acute phase response mediators [6668]. In response to many kinds of stress, cells produce heat shock proteins (HSPs), and it is believed that PDT can induce the cell surface expression and release of HSPs, which in turn stimulates the inflammatory and immune responses [69]. The body recognizes PDT-inflicted tumor tissue injury, and this further provokes a strong host response with neutrophilia as one of its manifestations [67]. In a rhabdomyosarcoma-bearing rat model, de Vree et al. [70] showed that PDT resulted in an increase in circulating neutrophils and the slowing of tumor growth. Depletion of neutrophils decreased the PDT-mediated effect on tumor growth. Antitumor immunity depends upon the presence of activated antigen presenting cells (APCs). PDT can increase the activity of APC and stimulate T-cell proliferation and T-cell secretion of interferon gamma [71]. The complement system is a biochemical cascade that consists of more than 30 serum and cell surface proteins [72]. The activated complement system was identified as an important element of the host response elicited by PDT [73, 74]. The complement system not only acts as a direct mediator of inflammation, but it also stimulates at least a dozen secondary inflammatory molecules, such as cytokines, interleukin-1beta (IL-1beta), TNF-alpha, IL-6, IL-10, granulocyte colony-stimulating factor, thromboxane, prostaglandins, leukotrienes, histamine, and coagulation factors [75]. Mroz et al. [76] recently showed that an effective vascular PDT regimen that can reliably promote local tumor destruction can also lead to antigen specific antitumor immunity. This tumor-destructive effect was mediated by tumor antigen-specific cytotoxic T cells. Moreover, PDT combined with low-dose cyclophosphamide can produce tumor-specific cytotoxic $\mathrm{T}$ cells and potent memory immunity, which in turn cause a dramatic improvement in survival and remission rates in a highly metastatic mouse tumor model [77] (Figure 5).

\section{Clinical Studies}

The first clinical trial of PDT in the treatment of locally advanced PC took place in 2002 [78]. In this phase I study, 16 inoperable patients with cancer in the head of the pancreas were treated with $\mathrm{mTHPC}(0.15 \mathrm{mg} / \mathrm{kg})$. After 3 days, light was delivered to the cancer percutaneously using fibers positioned under ultrasound or computerized tomographic guidance. The results showed that all patients had a new nonenhancing area in the pancreas consistent with tumor necrosis (range, $9.0-60.0 \mathrm{~cm}^{3}$ ), and the median survival time after PDT was 9.5 months (range, 4-30). PDT may be valuable for treating localized cancers in patients who are poor candidates for definitive surgery or in whom the location of the tumor makes pancreatic resection inappropriate. Abulafi et al. [79] and Tseng et al. [80] indicated that patients with pancreatic and ampullary carcinoma for whom surgery is not appropriate should be treated with PDT, which is both feasible and safe for small tumors.

Verteporfin, a derivative of a benzoporphyrin, has been proposed for the treatment of PC due to its short metabolic half-life, excitation by near infrared wavelengths and clinical approval for PDT [81]. Only 3 verteporfin PDT studies for PC have been undertaken. Ayaru et al. [82] concluded that the safety profile of verteporfin is very similar to mTHPC, with the advantages of a shorter drug-light interval and drug elimination time. Yusuf et al. [83] showed that endoscopic 


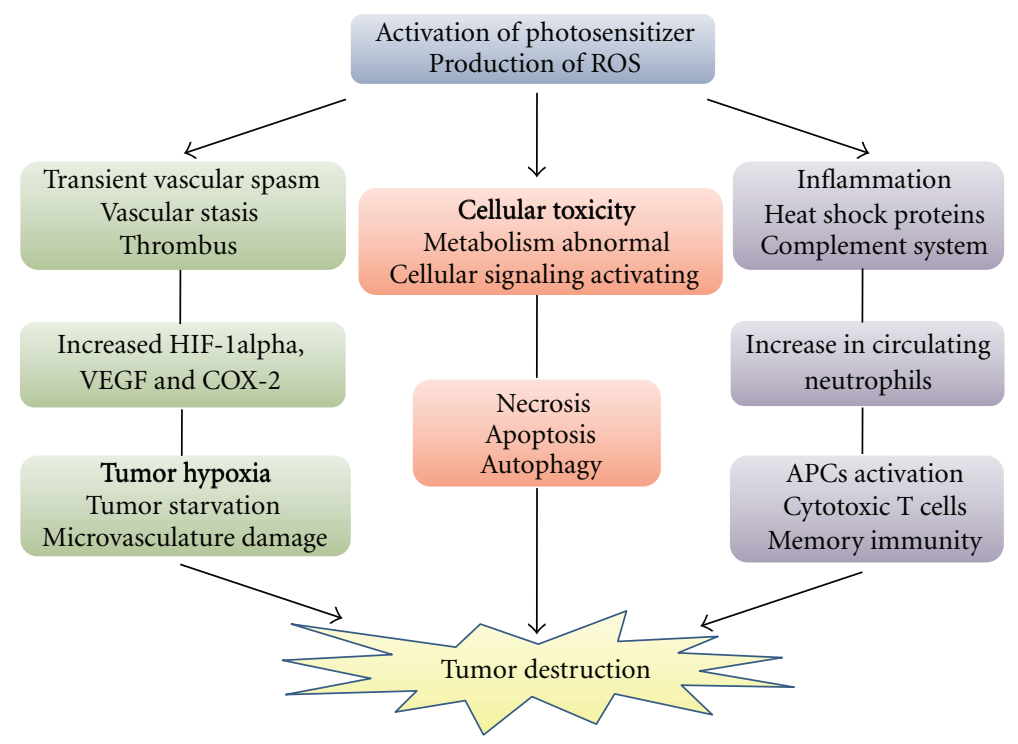

FIGURE 5: Pathways of PDT-induced pancreatic tumor cell death or destruction. The antitumor effects of PDT include three main mechanisms: direct tumor cell killing, vascular destruction, and immune system activation.

ultrasound- (EUS-) guided PDT of porcine pancreas with verteporfin led to pancreatic tissue damage. In different orthotopic PC xenograft models, Samkoe et al. [21] reported that both Panc-1 tumor and AsPC-1 tumor cells were killed by treatment with verteporfin PDT. Verteporfin PDT is now entering a Phase I/II clinical trial at the University College London Hospital [82].

Surgery remains the primary method of treatment for malignancies. However, more than $80 \%$ of PC patients have locally advanced or metastatic disease and thus are not amenable for resection at the time of diagnosis [3]. PDT has great potential when combined with surgical resection in the eradication of residual malignant tissues [84]. Surgical resection can clean the tumor bed within the obvious delineation while PDT may destroy the peripheral tissue and in turn enhance the efficiency of cancer removal [85].

PDT has many advantages, including its selective effect on malignant cancer cells of the pancreas versus normal tissue [18]. The precise reason for this phenomenon is still unclear, but it has been assumed to be related to an immunologic reaction [86]. Another advantage is that PDT does not lead to the accumulation of toxicity in patients $[7,87]$. Furthermore, the combination of PDT with chemotherapy and other treatments can lead to significant additive benefits [22]. However, PDT also has some disadvantages. Side effects such as gastrointestinal bleeding and duodenal obstruction [78] have been noted. In addition, large tumor masses prevent PDT from penetrating the full depth of the cancerous tissue and thus diminish its efficiency [88].

\section{Conclusions and Future Perspectives}

PC remains one of the most devastating neoplasms of the gastrointestinal tract. New therapeutic tools for PC are urgently needed. It is generally believed that the most common therapies for cancer, such as surgery, chemotherapy, and ionizing radiation, are accompanied by immuno-suppression. However, PDT does not share this characteristic and thus presents an attractive alternative to these therapies [65]. PDT in combination with surgery, radiotherapy, chemotherapy, or antiangiogenic therapy has become a subject of research in recent years. This strategy still faces challenges, such as the reduction of side effects and the optimization of the method of treatment (i.e., multiple interstitial optical fibers to increase treated tumor volume), but it may become a superior method for treating PC. Another way to improve PDT is through the development of new photosensitizers. Well-designed experimental studies and clinical studies will be needed for further improving PDT.

\section{Acknowledgments}

This study was supported by grants from the National Natural Science Foundation (Grant serial no. 30900705), 13115 Major Project (2010ZDKG-49), Scientific Grant of Xi'an City (2009 No.SF09027), and ND EPSCoR funds.

\section{References}

[1] A. Jemal, R. Siegel, J. Xu, and E. Ward, "Cancer statistics, 2010," CA Cancer Journal for Clinicians, vol. 60, no. 5, pp. 277$300,2010$.

[2] L. Herszényi and Z. Tulassay, "Epidemiology of gastrointestinal and liver tumors," European Review for Medical and Pharmacological Sciences, vol. 14, no. 4, pp. 249-258, 2010.

[3] C. J. Wray, S. A. Ahmad, J. B. Matthews, and A. M. Lowy, "Surgery for pancreatic cancer: recent controversies and current practice," Gastroenterology, vol. 128, no. 6, pp. 1626-1641, 2005. 
[4] E. H. Castellanos, D. B. Cardin, and J. D. Berlin, "Treatment of early-stage pancreatic cancer," Oncology, vol. 25, no. 2, pp. 182-189, 2011.

[5] C. J. Campen, T. Dragovich, and A. F. Baker, "Management strategies in pancreatic cancer," American Journal of HealthSystem Pharmacy, vol. 68, no. 7, pp. 573-584, 2011.

[6] C. M. Moore, D. Pendse, and M. Emberton, "Photodynamic therapy for prostate cancer-a review of current status and future promise," Nature Clinical Practice Urology, vol. 6, no. 1, pp. 18-30, 2009.

[7] C. A. Robertson, D. H. Evans, and H. Abrahamse, "Photodynamic therapy (PDT): a short review on cellular mechanisms and cancer research applications for PDT," Journal of Photochemistry and Photobiology B, vol. 96, no. 1, pp. 1-8, 2009.

[8] M. Alexiades-Armenakas, "Laser-mediated photodynamic therapy," Clinics in Dermatology, vol. 24, no. 1, pp. 16-25, 2006.

[9] B. Zhao and Y. Y. He, "Recent advances in the prevention and treatment of skin cancer using photodynamic therapy," Expert Review of Anticancer Therapy, vol. 10, no. 11, pp. 1797-1809, 2010.

[10] W. Jerjes, T. Upile, S. Akram, and C. Hopper, "The surgical palliation of advanced head and neck cancer using photodynamic therapy," Clinical Oncology, vol. 22, no. 9, pp. 785-791, 2010.

[11] M. A. Wildeman, H. J. Nyst, B. Karakullukcu, and B. I. Tan, "Photodynamic therapy in the therapy for recurrent/persistent nasopharyngeal cancer," Head \& Neck Oncology, vol. 1, article 40, 2009.

[12] M. Chen, A. Pennathur, and J. D. Luketich, "Role of photodynamic therapy in unresectable esophageal and lung cancer," Lasers in Surgery and Medicine, vol. 38, no. 5, pp. 396-402, 2006.

[13] L. Ayaru, S. G. Bown, and S. P. Pereira, "Photodynamic therapy for pancreatic and biliary tract carcinoma," International Journal of Gastrointestinal Cancer, vol. 35, no. 1, pp. 1-13, 2005.

[14] S. Jain and R. C. Kockelbergh, "The role of photodynamic diagnosis in the contemporary management of superficial bladder cancer," BJU International, vol. 96, no. 1, pp. 17-21, 2005.

[15] J. C. Stockert, M. Cañete, A. Juarranz et al., "Porphycenes: Facts and prospects in photodynamic therapy of cancer," Current Medicinal Chemistry, vol. 14, no. 9, pp. 997-1026, 2007.

[16] I. E. Kochevar and R. W. Redmond, "Photosensitized production of singlet oxygen," Methods in Enzymology, vol. 319, pp. 20-28, 2000.

[17] H. Kolarova, P. Nevrelova, K. Tomankova, P. Kolar, R. Bajgar, and J. Mosinger, "Production of reactive oxygen species after photodynamic therapy by porphyrin sensitizers," General Physiology and Biophysics, vol. 27, no. 2, pp. 101-105, 2008.

[18] B. G. Fan and A. Andren-Sandberg, "Photodynamic therapy for pancreatic cancer," Pancreas, vol. 34, no. 4, pp. 385-389, 2007.

[19] D. Kessel, M. G. H. Vicente, and J. J. Reiners, "Initiation of apoptosis and autophagy by photodynamic therapy," Lasers in Surgery and Medicine, vol. 38, no. 5, pp. 482-488, 2006.

[20] G. Kroemer, L. Galluzzi, P. Vandenabeele et al., "Classification of cell death: Recommendations of the Nomenclature Committee on Cell Death 2009," Cell Death and Differentiation, vol. 16, no. 1, pp. 3-11, 2009.

[21] K. S. Samkoe, A. Chen, I. Rizvi et al., "Imaging tumor variation in response to photodynamic therapy in pancreatic cancer xenograft models," International Journal of Radiation Oncology Biology Physics, vol. 76, no. 1, pp. 251-259, 2010.

[22] Q. Xie, L. Jia, Y. H. Liu, and C. G. Wei, "Synergetic anticancer effect of combined gemcitabine and photodynamic therapy on pancreatic cancer in vivo," World Journal of Gastroenterology, vol. 15, no. 6, pp. 737-741, 2009.

[23] J. Regula, B. Ravi, J. Bedwell, A. J. MacRobert, and S. G. Bown, "Photodynamic therapy using 5-aminolaevulinic acid for experimental pancreatic cancer-prolonged animal survival," British Journal of Cancer, vol. 70, no. 2, pp. 248-254, 1994.

[24] P. Mlkvy, H. Messman, A. J. MacRobert et al., "Photodynamic therapy of a transplanted pancreatic cancer model using meta-tetrahydroxyphenylchlorin (mTHPC)," British Journal of Cancer, vol. 76, no. 6, pp. 713-718, 1997.

[25] A. Hajri, S. Coffy, F. Vallat, S. Evrard, J. Marescaux, and M. Aprahamian, "Human pancreatic carcinoma cells are sensitive to photodynamic therapy in vitro and in vivo," British Journal of Surgery, vol. 86, no. 7, pp. 899-906, 1999.

[26] A. Ketabchi, A. MacRobert, P. M. Speight, and J. H. Bennett, "Induction of apoptotic cell death by photodynamic therapy in human keratinocytes," Archives of Oral Biology, vol. 43, no. 2, pp. 143-149, 1998.

[27] C. S. M. Ferreira, M. C. Cheung, S. Missailidis, S. Bisland, and J. Gariépy, "Phototoxic aptamers selectively enter and kill epithelial cancer cells," Nucleic Acids Research, vol. 37, no. 3, pp. 866-876, 2009.

[28] M. Blank and Y. Shiloh, "Programs for cell death: apoptosis is only one way to go," Cell Cycle, vol. 6, no. 6, pp. 686-695, 2007.

[29] J. Usuda, T. Hirata, S. Ichinose et al., "Tailor-made approach to photodynamic therapy in the treatment of cancer based on Bcl-2 photodamage," International Journal of Oncology, vol. 33, no. 4, pp. 689-696, 2008.

[30] R. Z. Renno, F. C. Delori, R. A. Holzer, E. S. Gragoudas, and J. W. Miller, "Photodynamic therapy using Lu-Tex induces apoptosis in vitro, and its effect is potentiated by angiostatin in retinal capillary endothelial cells," Investigative Ophthalmology and Visual Science, vol. 41, no. 12, pp. 3963-3971, 2000.

[31] J. He, M. L. Agarwal, H. E. Larkin, L. R. Friedman, L. Y. Xue, and N. L. Oleinick, "The Induction of Partial Resistance to Photodynamic Therapy by the Protooncogene BCL-2," Photochemistry and Photobiology, vol. 64, no. 5, pp. 845-852, 1996.

[32] X. Chen, P. Zhao, F. Chen, L. Li, and R. Luo, "Effect and mechanism of 5-aminolevulinic acid-mediated photodynamic therapy in esophageal cancer," Lasers in Medical Science, vol. 26, no. 1, pp. 69-78, 2010.

[33] C. M. N. Yow, N. K. Mak, A. W. N. Leung, and Z. Huang, "Induction of early apoptosis in human nasopharyngeal carcinoma cells by mTHPC-mediated photocytotoxicity," Photodiagnosis and Photodynamic Therapy, vol. 6, no. 2, pp. 122-127, 2009.

[34] Z. W. Liu, Y. P. Zhao, Q. Liao, Y. D. Wu, and Y. Zhu, "Relationship between apoptosis induced by 2-butylamino-2demethoxy-hypocrellin B in human pancreatic cancer cells Capan-1 and photosensitization of mitochondria," Zhonghua Wai Ke Za Zhi, vol. 44, no. 1, pp. 36-39, 2006.

[35] D. Kessel and Y. Luo, "Photodynamic therapy: a mitochondrial inducer of apoptosis," Cell Death and Differentiation, vol. 6, no. 1, pp. 28-35, 1999.

[36] Y. -M. Jeong, H. Li, S. Y. Kim et al., "Photo-activated 5hydroxyindole-3-acetic acid induces apoptosis of prostate and bladder cancer cells," Journal of Photochemistry and Photobiology B, vol. 103, no. 1, pp. 50-56, 2011. 
[37] D. I. O’Brien, K. Nally, R. G. Kelly, T. M. O’Connor, F. Shanahan, and J. O'Connell, "Targeting the Fas/Fas ligand pathway in cancer," Expert Opinion on Therapeutic Targets, vol. 9, no. 5, pp. 1031-1044, 2005.

[38] M. Olivo and M. Ali-Seyed, "Apoptosis signalling mechanisms in human cancer cells induced by Calphostin-PDT," International Journal of Oncology, vol. 30, no. 3, pp. 537-548, 2007.

[39] O. Inanami, A. Yoshito, K. Takahashi, W. Hiraoka, and M. Kuwabara, "Effects of BAPTA-AM and forskolin on apoptosis and cytochrome $\mathrm{c}$ release in photosensitized Chinese hamster V79 cells," Photochemistry and Photobiology, vol. 70, no. 4, pp. 650-655, 1999.

[40] J. F. Chiou, Y. H. Wang, M. J. Jou, T. Z. Liu, and C. Y. Shiau, "Verteporfin-photoinduced apoptosis in HepG2 cells mediated by reactive oxygen and nitrogen species intermediates," Free Radical Research, vol. 44, no. 2, pp. 155-170, 2010.

[41] R. D. Almeida, B. J. Manadas, A. P. Carvalho, and C. B. Duarte, "Intracellular signaling mechanisms in photodynamic therapy," Biochimica et Biophysica Acta, vol. 1704, no. 2, pp. 59-86, 2004.

[42] S. Galaz, J. Espada, J. C. Stockert et al., "Loss of E-cadherin mediated cell-cell adhesion as an early trigger of apoptosis induced by photodynamic treatment," Journal of Cellular Physiology, vol. 205, no. 1, pp. 86-96, 2005.

[43] C. Volanti, G. Gloire, A. Vanderplasschen, N. Jacobs, Y. Habraken, and J. Piette, "Downregulation of ICAM-1 and VCAM-1 expression in endothelial cells treated by photodynamic therapy," Oncogene, vol. 23, no. 53, pp. 8649-8658, 2004.

[44] E. Buytaert, M. Dewaele, and P. Agostinis, "Molecular effectors of multiple cell death pathways initiated by photodynamic therapy," Biochimica et Biophysica Acta, vol. 1776, no. 1, pp. 86-107, 2007.

[45] J. Zawacka-Pankau, J. Krachulec, I. Grulkowski, K. P. Bielawski, and G. Selivanova, "The p53-mediated cytotoxicity of photodynamic therapy of cancer: recent advances," Toxicology and Applied Pharmacology, vol. 232, no. 3, pp. 487-497, 2008.

[46] M. Mitsunaga, A. Tsubota, K. Nariai et al., "Early apoptosis and cell death induced by ATX-S10Na (II)-mediated photodynamic therapy are Bax- and p53-dependent in human colon cancer cells," World Journal of Gastroenterology, vol. 13, no. 5, pp. 692-698, 2007.

[47] D. S. Lim, S. M. Bae, S. Y. Kwak et al., "Adenovirus-mediated p53 treatment enhances photodynamic antitumor response," Human Gene Therapy, vol. 17, no. 3, pp. 347-352, 2006.

[48] B. W. Jia and X. L. Lian, "Use of photodynamic therapy in malignant lesions of stomach, bile duct, pancreas, colon and rectum," Hepato-Gastroenterology, vol. 54, no. 75, pp. 718724, 2007.

[49] T. Yorimitsu and D. J. Klionsky, "Autophagy: molecular machinery for self-eating," Cell Death and Differentiation, vol. 12, no. 2, pp. 1542-1552, 2005.

[50] G. Kroemer and M. Jäättelä, "Lysosomes and autophagy in cell death control," Nature Reviews Cancer, vol. 5, no. 11, pp. 886897, 2005.

[51] G. Kroemer, W. S. El-Deiry, P. Golstein et al., "Classification of cell death: recommendations of the Nomenclature Committee on Cell Death," Cell Death and Differentiation, vol. 12, no. 2, pp. 1463-1467, 2005.

[52] J. J. Reiners Jr., P. Agostinis, K. Berg, N. L. Oleinick, and D. Kessel, "Assessing autophagy in the context of photodynamic therapy," Autophagy, vol. 6, no. 1, pp. 7-18, 2010.
[53] A. Criollo, M. C. Maiuri, E. Tasdemir et al., "Regulation of autophagy by the inositol trisphosphate receptor," Cell Death and Differentiation, vol. 14, no. 5, pp. 1029-1039, 2007.

[54] A. Weyergang, K. Berg, O. Kaalhus, Q. Peng, and P. K. Selbo, "Photodynamic therapy targets the mTOR signaling network in Vitro and in Vivo," Molecular Pharmaceutics, vol. 6, no. 1, pp. 255-264, 2009.

[55] D. Kessel and A. S. Arroyo, "Apoptotic and autophagic responses to Bcl-2 inhibition and photodamage," Photochemical and Photobiological Sciences, vol. 6, no. 12, pp. 1290-1295, 2007.

[56] P. Carmeliet and R. K. Jain, "Angiogenesis in cancer and other diseases," Nature, vol. 407, no. 6801, pp. 249-257, 2000.

[57] V. H. Fingar, T. J. Wieman, S. A. Wiehle, and P. B. Cerrito, "The role of microvascular damage in photodynamic therapy: the effect of treatment on vessel constriction, permeability, and leukocyte adhesion," Cancer Research, vol. 52, no. 18, pp. 4914-4921, 1992.

[58] J. H. Woodhams, L. Kunz, S. G. Bown, and A. J. MacRobert, "Correlation of real-time haemoglobin oxygen saturation monitoring during photodynamic therapy with microvascular effects and tissue necrosis in normal rat liver," British Journal of Cancer, vol. 91, no. 4, pp. 788-794, 2004.

[59] L. B. Li and R. C. Luo, "Effect of drug-light interval on the mode of action of Photofrin photodynamic therapy in a mouse tumor model," Lasers in Medical Science, vol. 24, no. 4, pp. 597-603, 2009.

[60] R. Bhuvaneswari, G. Y. Yuen, S. K. Chee, and M. Olivo, "Hypericin-mediated photodynamic therapy in combination with Avastin (bevacizumab) improves tumor response by downregulating angiogenic proteins," Photochemical and Photobiological Sciences, vol. 6, no. 12, pp. 1275-1283, 2007.

[61] Q. Zhou, M. Olivo, K. Y. K. Lye, S. Moore, A. Sharma, and B. Chowbay, "Enhancing the therapeutic responsiveness of photodynamic therapy with the antiangiogenic agents SU5416 and SU6668 in murine nasopharyngeal carcinoma models," Cancer Chemotherapy and Pharmacology, vol. 56, no. 6, pp. 569-577, 2005.

[62] R. Bhuvaneswari, Y. Y. Y. Gan, K. K. L. Yee, K. C. Soo, and M. Olivo, "Effect of hypericin-mediated photodynamic therapy on the expression of vascular endothelial growth factor in human nasopharyngeal carcinoma," International Journal of Molecular Medicine, vol. 20, no. 4, pp. 421-428, 2007.

[63] J. Reyal, "Arterial flow focalization could increase tissue oxygen partial pressure, or trigger endothelial shear stress-a new concept to overcome cancer hypoxia-induced radiotherapy resistance, or stimulate liver regeneration during fulminant hepatitis," Medical Hypotheses, vol. 74, no. 2, pp. 301-308, 2010.

[64] B. W. Henderson and T. J. Dougherty, "How does photodynamic therapy work?" Photochemistry and Photobiology, vol. 55, no. 1, pp. 145-157, 1992.

[65] M. L. Agarwal, H. E. Larkin, S. I. A. Zaidi, H. Mukhtar, and N. L. Oleinick, "Phospholipase activation triggers apoptosis in photosensitized mouse lymphoma cells," Cancer Research, vol. 53, no. 24, pp. 5897-5902, 1993.

[66] S. O. Gollnick, S. S. Evans, H. Baumann et al., "Role of cytokines in photodynamic therapy-induced local and systemic inflammation," British Journal of Cancer, vol. 88, no. 11, pp. 1772-1779, 2003.

[67] I. Cecic, B. Stott, and M. Korbelik, "Acute phase responseassociated systemic neutrophil mobilization in mice bearing tumors treated by photodynamic therapy," International Immunopharmacology, vol. 6, no. 8, pp. 1259-1266, 2006. 
[68] M. Korbelik, I. Cecic, S. Merchant, and J. Sun, "Acute phase response induction by cancer treatment with photodynamic therapy," International Journal of Cancer, vol. 122, no. 6, pp. 1411-1417, 2008.

[69] M. Korbelik, J. Sun, and I. Cecic, "Photodynamic therapyinduced cell surface expression and release of heat shock proteins: relevance for tumor response," Cancer Research, vol. 65, no. 3, pp. 1018-1026, 2005.

[70] W. J. A. de Vree, M. C. Essers, H. S. de Bruijn, W. M. Star, J. F. Koster, and W. Sluiter, "Evidence for an important role of neutrophils in the efficacy of photodynamic therapy in vivo," Cancer Research, vol. 56, no. 13, pp. 2908-2911, 1996.

[71] S. O. Gollnick, B. Owczarczak, and P. Maier, "Photodynamic therapy and anti-tumor immunity," Lasers in Surgery and Medicine, vol. 38, no. 5, pp. 509-515, 2006.

[72] M. J. Walport, "Advances in immunology: complement (first of two parts)," The New England Journal of Medicine, vol. 344, no. 14, pp. 1058-1066, 2001.

[73] B. Stott and M. Korbelik, "Activation of complement C3, C5, and C9 genes in tumors treated by photodynamic therapy," Cancer Immunology, Immunotherapy, vol. 56, no. 5, pp. 649658, 2007.

[74] M. Korbelik and I. Cecic, "Complement activation cascade and its regulation: relevance for the response of solid tumors to photodynamic therapy," Journal of Photochemistry and Photobiology B, vol. 93, no. 1, pp. 53-59, 2008.

[75] I. Cecic and M. Korbelik, "Mediators of peripheral blood neutrophilia induced by photodynamic therapy of solid tumors," Cancer Letters, vol. 183, no. 1, pp. 43-51, 2002.

[76] P. Mroz, A. Szokalska, M. X. Wu, and M. R. Hamblin, "Photodynamic therapy of tumors can lead to development of systemic antigen-specific immune response," PLoS ONE, vol. 5, no. 12, Article ID e15194, 2010.

[77] A. P. Castano, P. Mroz, M. X. Wu, and M. R. Hamblin, "Photodynamic therapy plus low-dose cyclophosphamide generates antitumor immunity in a mouse model," Proceedings of the National Academy of Sciences of the United States of America, vol. 105, no. 14, pp. 5495-5500, 2008.

[78] S. G. Bown, A. Z. Rogowska, D. E. Whitelaw et al., "Photodynamic therapy for cancer of the pancreas," Gut, vol. 50, no. 4, pp. 549-557, 2002.

[79] A. M. Abulafi, J. T. Allardice, N. S. Williams, N. Van Someren, C. P. Swain, and C. Ainley, "Photodynamic therapy for malignant tumours of the ampulla of Vater," Gut, vol. 36, no. 6, pp. 853-856, 1995.

[80] W. W. Tseng, R. E. Saxton, A. Deganutti, and C. D. Liu, "Infrared laser activation of indocyanine green inhibits growth in human pancreatic cancer," Pancreas, vol. 27, no. 3, pp. e42e45, 2003.

[81] Z. Huang, "A review of progress in clinical photodynamic therapy," Technology in Cancer Research and Treatment, vol. 4, no. 3, pp. 283-293, 2005.

[82] L. Ayaru, J. Wittmann, A. J. MacRobert, M. Novelli, S. G. Bown, and S. P. Pereira, "Photodynamic therapy using verteporfin photosensitization in the pancreas and surrounding tissues in the Syrian golden hamster," Pancreatology, vol. 7, no. 1, pp. 20-27, 2007.

[83] T. E. Yusuf, K. Matthes, and W. R. Brugge, "EUS-guided photodynamic therapy with verteporfin for ablation of normal pancreatic tissue: a pilot study in a porcine model (with video)," Gastrointestinal Endoscopy, vol. 67, no. 6, pp. 957-961, 2008.
[84] H. I. Pass, T. F. DeLaney, Z. Tochner et al., "Intrapleural photodynamic therapy: results of a phase I trial," Annals of Surgical Oncology, vol. 1, no. 1, pp. 28-37, 1994.

[85] T. Momma, M. R. Hamblin, H. C. Wu, and T. Hasan, "Photodynamic therapy of orthotopic prostate cancer with benzoporphyrin derivative: local control and distant metastasis," Cancer Research, vol. 58, no. 23, pp. 5425-5431, 1998.

[86] P. J. O. Nuutinen, P. T. Chatlani, J. Bedwell, A. J. MacRobert, D. Phillips, and S. G. Bown, "Distribution and photodynamic effect of disulphonated aluminium phthalocyanine in the pancreas and adjacent tissues in the Syrian golden hamster," British Journal of Cancer, vol. 64, no. 6, pp. 1108-1115, 1991.

[87] F. S. De Rosa and M. V. L. B. Bentley, "Photodynamic therapy of skin cancers: sensitizers, clinical studies and future directives," Pharmaceutical Research, vol. 17, no. 12, pp. 1447-1455, 2000.

[88] B. C. Wilson, "Potential applications of photodynamic therapy in regenerative medicine," The Journal of Craniofacial Surgery, vol. 14, no. 3, pp. 278-283, 2003. 


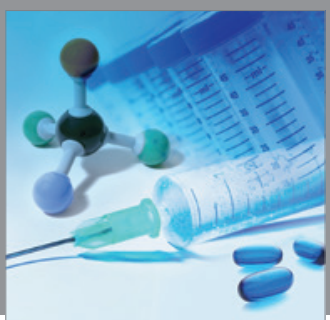

International Journal of

Medicinal Chemistry

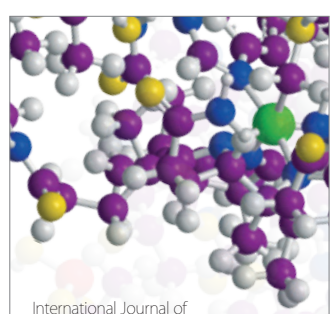

Carbohydrate Chemistry

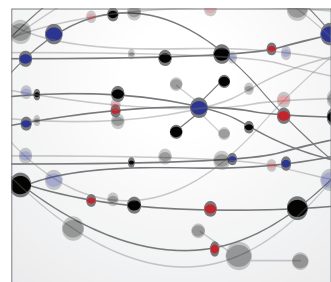

The Scientific World Journal
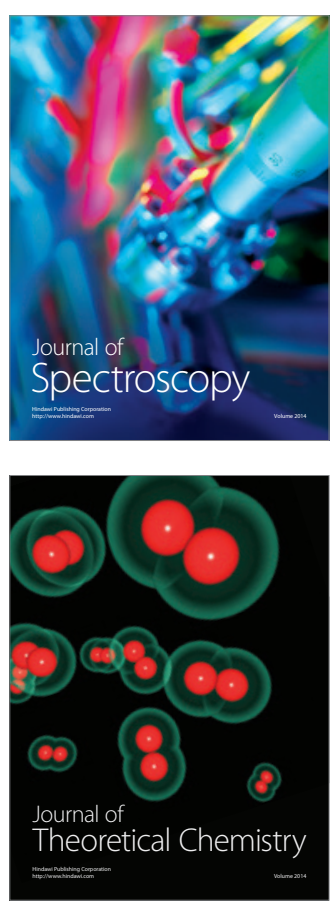
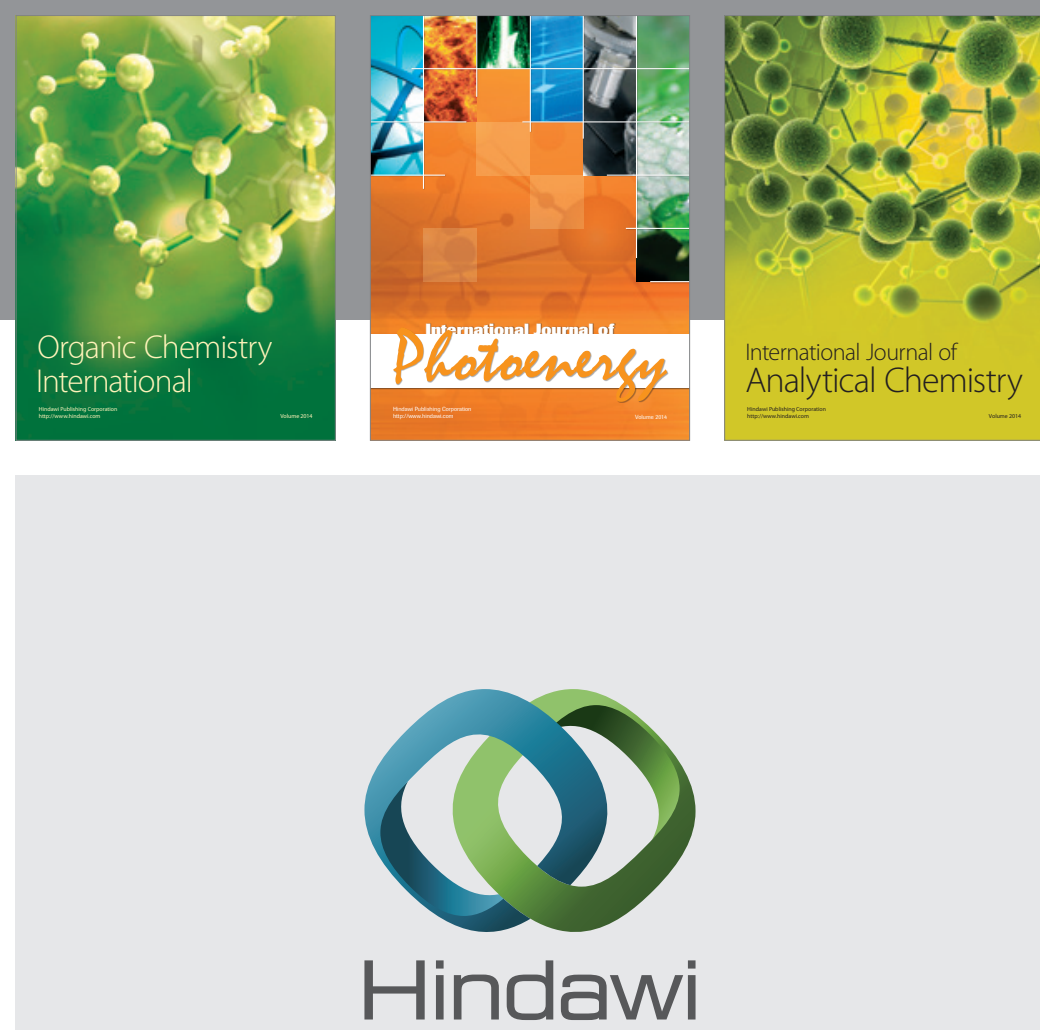

Submit your manuscripts at

http://www.hindawi.com
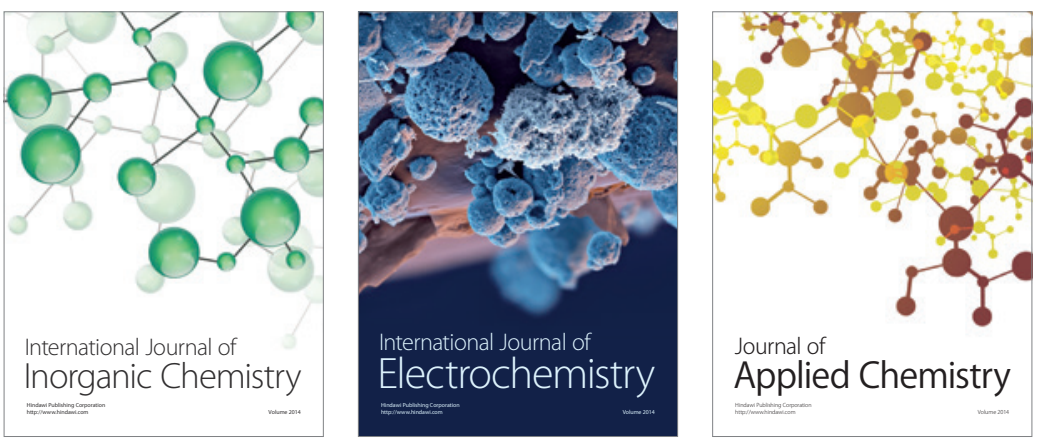

Journal of

Applied Chemistry
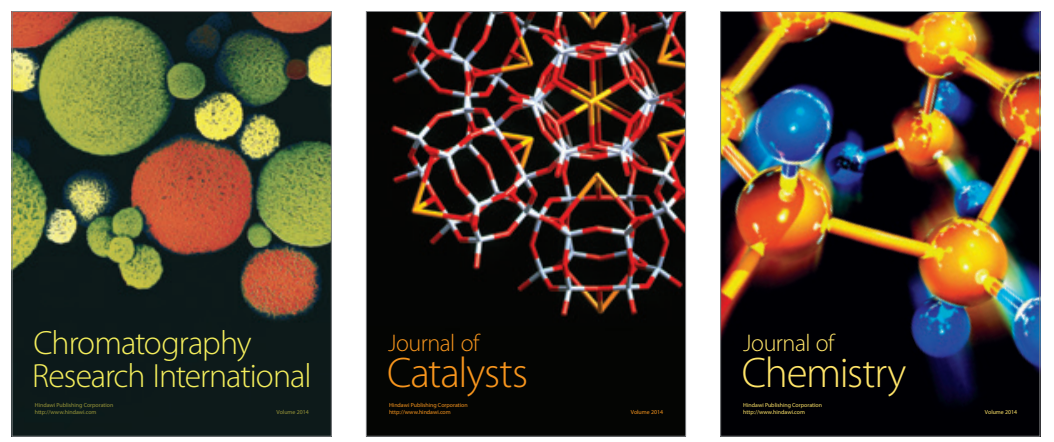
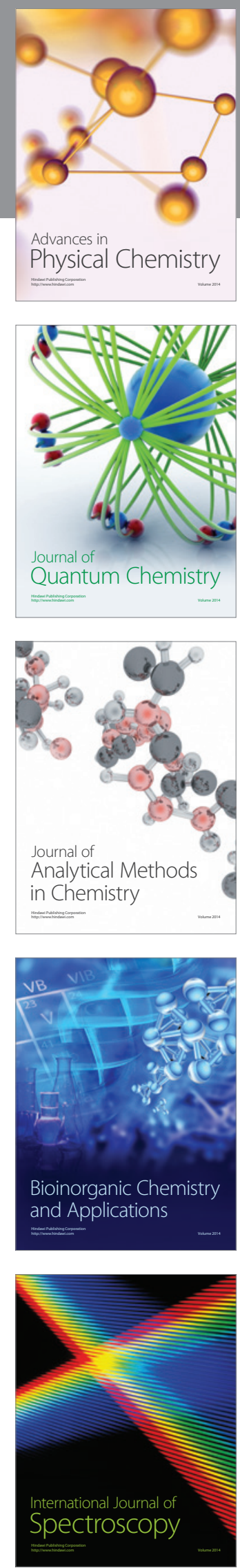